\title{
PERFORMANCE ANALYSIS OF MPLS AND TRADITIONAL IP IN NODE SCALABLE NETWORKS
}

\author{
${ }^{1}$ Muhammad Arshad, ${ }^{2}$ Lala Rukh, ${ }^{3}$ Hussain Shah, ${ }^{1}$ Bilal Khan, ${ }^{1}$ Nazir Jan, ${ }^{1}$ Shahzad Ali \\ ${ }^{1}$ City University of Science and Information Technology, Peshawar, Pakistan \\ ${ }^{2}$ Institute of Business and Management Sciences, University of Agriculture Peshawar, Pakistan \\ ${ }^{3}$ Shaykh zayed Islamic Centere, Unversity of Peshawar, Pakistan \\ 19arshad12@aup.edu.pk, ${ }^{2}$ rukh82@yahoo.com, ${ }^{3}$ hussainshahsafi@gmail.com, ${ }^{4}$ bilalsoft63@gmail.com, ${ }^{5}$ engr.nazirjan@gmail.com, \\ 'shahzad.ali@cusit.edu.pk
}

\begin{abstract}
Multiprotocol label switching is the latest and developing technology in the world of the internet. It speeds up the network by using the technique of label instead of an IP address. It provides the reliable transmission of data with high speed and low delay. For efficient use of network utilization MPLS has a key feature of QoS. Due to the effective utilization of network resources, minimum delay and predictable performance MPLS technology make it more appropriate for implementing multimedia type applications. In this research, the performance of MPLS technology is compared with the traditional IP network for multimedia traffic in node scalable networks. For simulating and comparing the performance of both technologies OPNET modular 14.5 is used. This comparison is done on the basis of network performance parameters such as packet loss/ traffic drop, end-to-end delay, and throughput. Finally, the results have been evaluated which show that MPLS technology provides better performance as compared to IP in node scalable environment.
\end{abstract}

Keywords-- Multiprotocol Label Switching, Traffic Engineering, Internet Protocol, Quality of Service, Optimized Network Engineering Tool.

\section{INTRODUCTION}

Nowadays, the use of the internet is more complex, large and due to this complexity of the services, internet face many challenges, like the service provider always have to upgrade their network to meet the requirement of the services. The services which are provided by the existence IP network are not more efficient for the modern world's application i.e real-time application, that's why there should be a technique that addresses the guaranteed delivery of the required services. The routing table of the existence IP network is very complex that's why it takes more processing time. While MPLS technology has the ability to transmit the information without the need of a dedicated line by using a label instead of an IP address [1].

While using MPLS which is a switching technology, incoming packets are labeled and forwarded according to their label within the MPLS network. These labels are usually matched to the destination path in the network layer or data link layer's circuit or edge router's outgoing interface. It works as an intermediate between the network and data link layer that's why it is sometimes referred as, 2.5 layer technology [2].

MPLS is a new technology deployed by ISP networks to tackle problems faced by the existing IP networks. MPLS is packet forwarding technology based on labeling, where labels are assigned to each IP packet and are then forwarded on the base of labels instead of the destination address [3].

\section{A) MPLS HEADER}

The header of MPLS, also known as SHIM header, consists of the following 4 parts [4] as shown in Figure 1.

Label value bit: The initial 20 bits are known as label bits and its range can be from 0 to 9,10 to 19 or we can say it can be 1048575 .

Experimental bit: These bits are used for the Qos and also for expressing the class of service.

Label stack bit This bit is an order set of the MPLS labels. It is set to 1 if the label is the last label and set to 0 for others.

TTL Time-to-live bit: With the help of these bits unwanted packets loop is discarded.

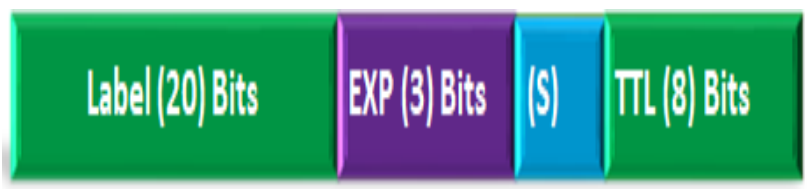

Fig (1) Syntax of MPLS Header [4]

\section{B) Internet Protocol (IP)}

In the IP network, each router has the ability to forward the data packets. In the initial phase, data link layer process will complete and after that, in the network layer, the entire routing table is checked for routing the packets based on the destination address. The layer 2 process will be in control plane of router.Each and every time for all those packets, 
which are on data plane, the router must repeat the above step to find out the next hop for the packets. For taking decision there is a transformation from control to data plane each time that seems very time consuming process [5].

\section{RELATED WORK}

Adaquate researche has been made by different researchers towards the above said problem of IP Networks. In the literature review, the researchers discussed the different performance measures of MPLS over traditional IP in different environments.

According to He and Botham (2008) [6] MPLS is not the replacement for conventional longest-match routing. It allows ISPs to design new services that can potentially generate revenue for them. Because of its superior capacity to provide virtual private network (VPN) and traffic engineering (TE) services, MPLS is now considered as an important technology for the management and operation of IP networks. MPLS makes routing decisions on the basis of the value of the label instead of destination IP address thus providing privacy and supports private and non-unique IP addresses within the VPN.

Rahman et al.(2008) [7] contended that the main functionality of the traffic engineering is asset reservation and ideal asset usage. Multi-protocol exchanging innovation permits the traffic engineering and increments the exhibition over the conventional IP network. In his exploration, an empirical study among existing IP network and MPLS has been done, as indicated by his examination it has been observed that MPLS has the competency to devour the current network bandwidth in a precisely capable manner to expand the enactment of the network.

Veni et al., (2010) [1] depicted that MPLS is, for the most part, utilized for the traffic engineering reason with in the ISP (Internet Service Provider) network. The center profit of the MPLS network, which is contrasted with the traditional IP routing, is that MPLS can basically and effectively sidestep the shortest path which is being chosen by the IP network or routing.

This procedure can be executed by making the label switch path between a couple of router within the network. MPLS is a convenient answer for comprehending the trouble which is tested by existing days network such is speed, Quality of Service (QoS) the management and traffic engineering.

Bhandure et al., (2013) [5] depicted that in the Internet Protocol (IP) network, each hop needs to rely on its own choice when the packet arrived at the network.In the beginning, the data link layer process will be finished through mac address and afterward, layer 3 processing will be completed which is in the control plane. In each stage for all packets, every router passes through the similar process in order to locate the following hop for a packet. The main issue with ordinary routing protocol is that for the impeccable end making process, there will be many times changes from the control plane to the data plane. So this is an intricate method. Just as IP routing is executed at each and every hop of the packet path in the network. The entire IP header investigation is finished at each router which is time squandering.

Mishra et al., (2014) [8] compared the performance of both MPLS and traditional IP network for VoIP application on the basis of performance metrics such as end to end delay and voice packet drop. According to this study, it is concluded that MPLS gives better results in the VoIP application.

Akshay and Pooja (2015) [3] compared the MPLS network with OSI model, on the basis of his study, MPLS network sometimes calleded 2.5 layer technology, because it operates between the data link layer and network layer. MPLS technology can be used for different types of data including sonnet, ATM and also for traditional IP packets.

Rani and Goyal (2016) [9] evaluated the usage of network resources by using the traffic engineering technique of MPLS. They evaluated performance comparison of both the technologies on basis of end to end delay, FTP response time and throughput, MPLS traffic engineering gives much better performance than the traditional IP in a dense/ heavy weighted network, i.e heavy traffic and also in multimedia traffic network.

Tahir et al., (2017) [10] expressed that the transmission of voice application over the internet is the fundamental need of each association on the planet. Numerous systems are executed for the dependable transmission of VoIP over the internet. In his study, a relative investigation of ordinary IP and MPLS have been accomplished for VoIP. Multi-protocol Label Switching is a quick and trustworthy innovation that makes the transmission of each kind of traffic mode superior to its counterpart IP. The VoIP traffic is considered to be the most vulnerable traffic to environmental noises that's why MPLS innovation is best suited. The MPLS based system forward VoIP application from sender to receiver with low postponement and packet lost by empowering QoS for different sorts of TCP and UDP traffic.

Katyar and Shrama (2017) [11] discussed the presentation enhancement of IP and MPLS network in VoIP application. The most recent advancement of IP network are using voice applications that are progressively perplexing and the bandwidth requirements for them will be high. In the ongoing decade, IP networks are utilizing MPLS technology to improve the exhibition of IP networks, in MPLS technology data packets can be treated based on labels rather than IP address. MPLS has different advantages like Traffic Engineering (TE), QoS and VPNs and so on. The focal highlights of MPLS are TE.

\section{III.Tools and Statistics}

Table 1 depicts number of routers, Simulation tools, 
Technology, Simulation time and other perameters involved in the simulation.

Table (1) Simulation Parameters

\begin{tabular}{|l|l|}
\hline \multicolumn{1}{|c|}{ Parameters } & \multicolumn{1}{c|}{ Description } \\
\hline Simulation tool & OPNET Modular 14.5 \\
\hline Technology used & IP and MPLS \\
\hline Simulation time & 10 minutes \\
\hline Number of Routers & $4,8,12$ \\
\hline Traffic type & Multimedia \\
\hline Area & $1000 \mathrm{~m}$ x $1000 \mathrm{~m}$ \\
\hline Protocols & LDP,OSPF \\
\hline Number of Stations/switches & 2 \\
\hline Number of End users & $10,50,100$ \\
\hline
\end{tabular}

\section{METHODOLOGY}

This Paper focuses on the performance of MPLS and Traditional IP network in a node scalable environment on the basis of Performance matrics that are Throughput [12], Delay and Packet Loss [13], All the simulations are carried out in OPNET Simulator 14.5. Figure 2 describes the methodology of this research.

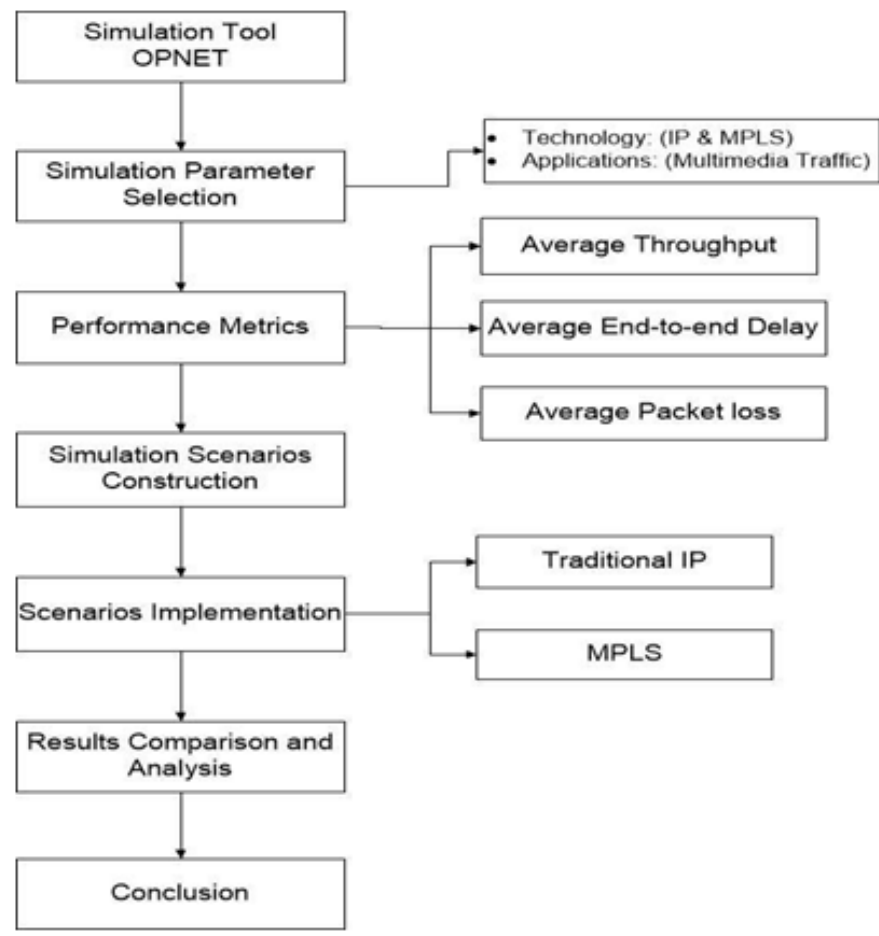

Fig (2) Flow Chart of Research Methodology

\section{A) Network Simulation Design}

A number of scenarios are simulated using the OPNET simulator to study and analyzing the performance of IP and MPLS networks in node scalable environment. The performance is measured on the basis of performance parameters i.e, End to End delay, throughput and packet loss.

In the scenario shown in Figure 3, four core routers and two edge routers are taken, further, there are two switches, ten (10) end-user and one server are taken. The end-user is connected with switch 1 and the server is connected with switch 2. For connectivity between the routers, DS1 links are used.The other two objects are application definition and profile definition. From application definition data traffic is set on the server and from profile definition the end-users are permitted to access that traffic.

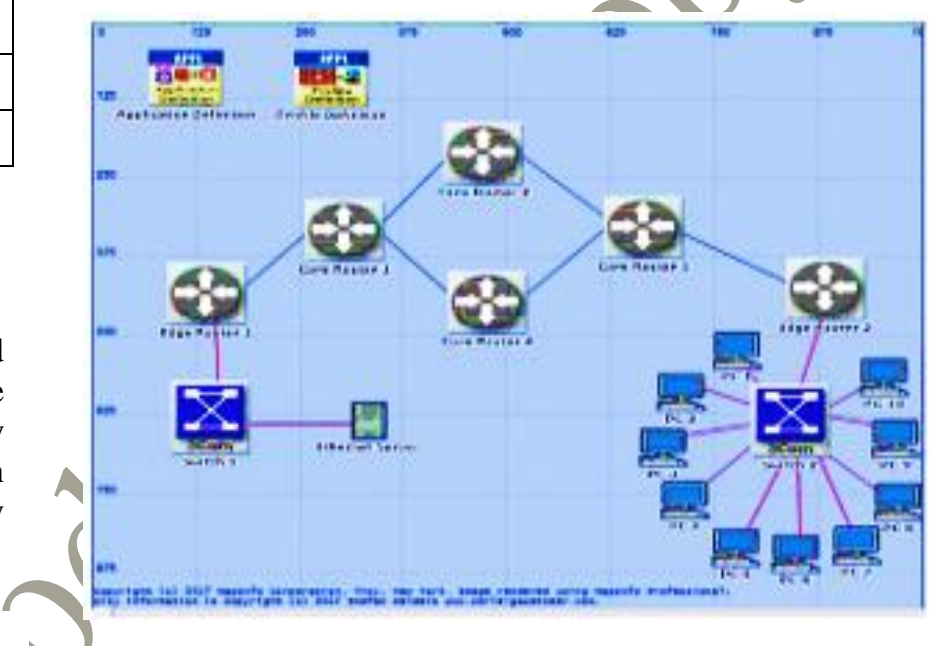

Fig (3) Layout of IP Simulation Model of Four Routers

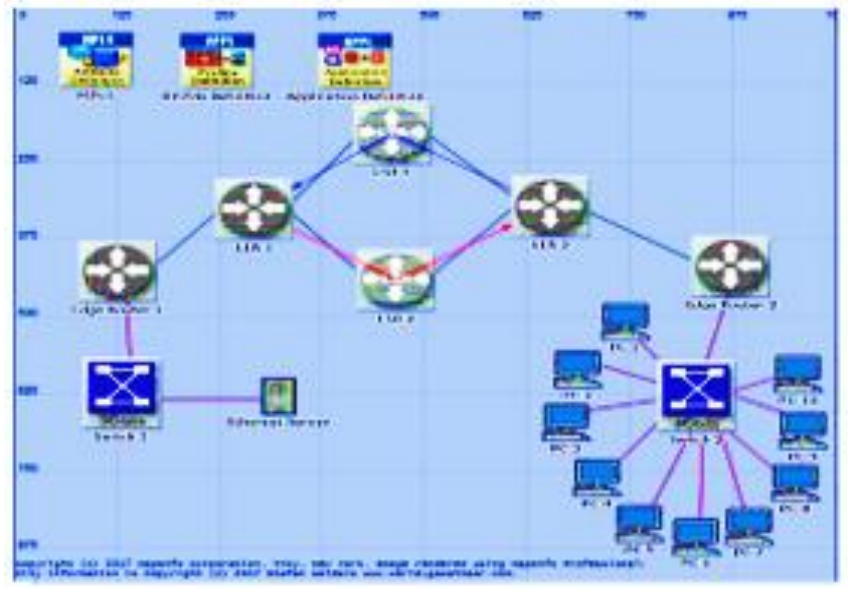

Fig (4) Layout of MPLS Simulation Model of Four Routers

Figure 4 scenario is same as Figure 3 of the IP network but with a little bit changes as there is one extra attribute added, as MPLS attribute definition. The core inner routers are replaced with LSR (Label switch router) while the core outer routers are replaced with LER (Label edge router) for the MPLS network, also LSP is configured in core routers. The functionality of LSR routers is the switching of label 
packets while LER is responsible for encapsulating or deencapsulating labels to packet when enter or leave the MPLS core network.

Figure 5 shows the second scenario of IP network the number of end-user changed from 10 to 50, also routers in the core network are increased as this research is focused on node scalable environment. There are four varying routers and workstations to analyzing the performance of the network.

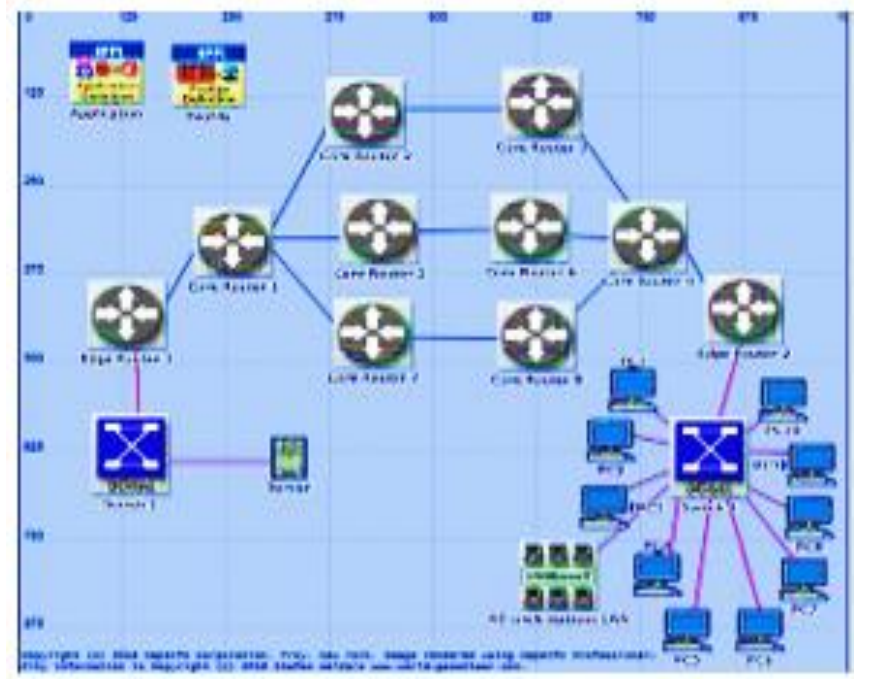

Fig (5) layout of IP Simulation Model of Eight Routers

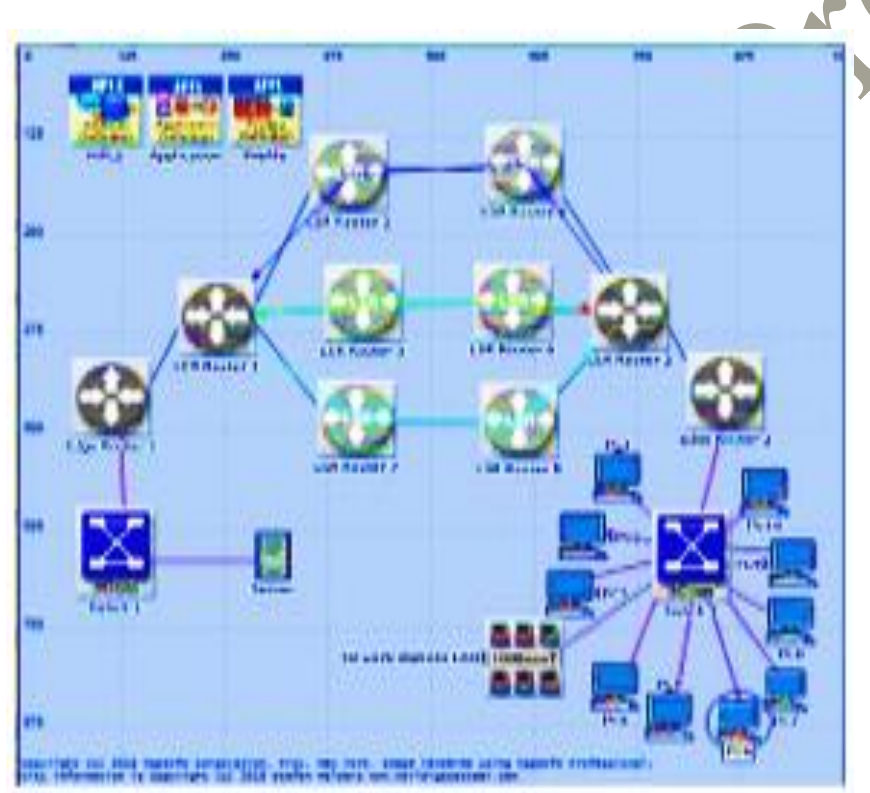

Fig (6) Layout of MPLS Simulation Model of Eight Routers

Figure 6 describes scenario 2 of the MPLS network with eight core routers. Router 1 and router 4 are replaced with LER router 1 and LER router 2, while the remaining 6 routers are replaced with LSR routers.

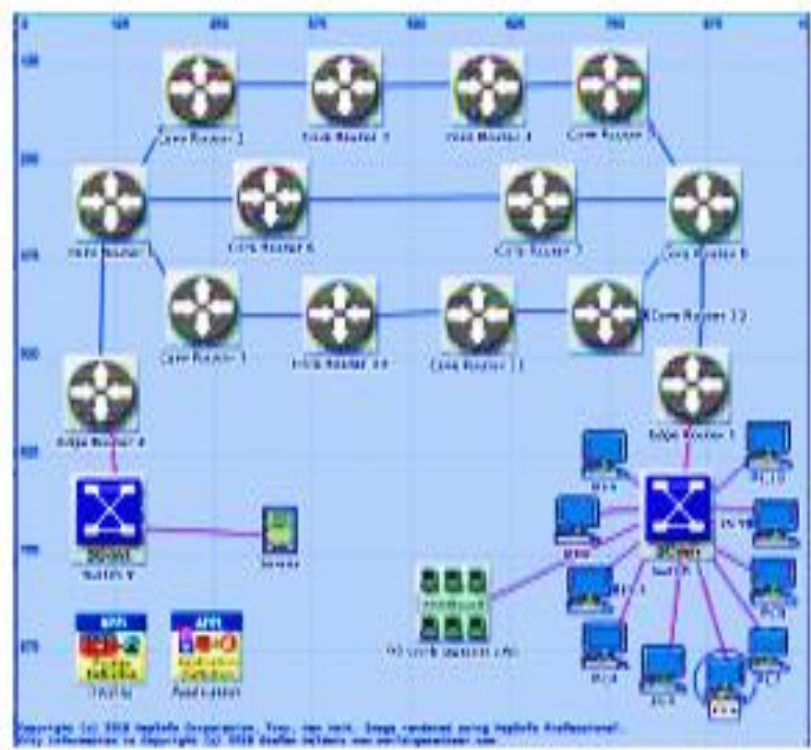

Fig (7) Layout of IP Simulation Model of Twelve Routers

Figure 7 represents scenario 3 of IP network for conducting this research as this has been discussed alreay that this research is focused on node scalability so for the purpose of achieving the goal of this research, No of node in each scenario are varying for comparing the performance. each scenary

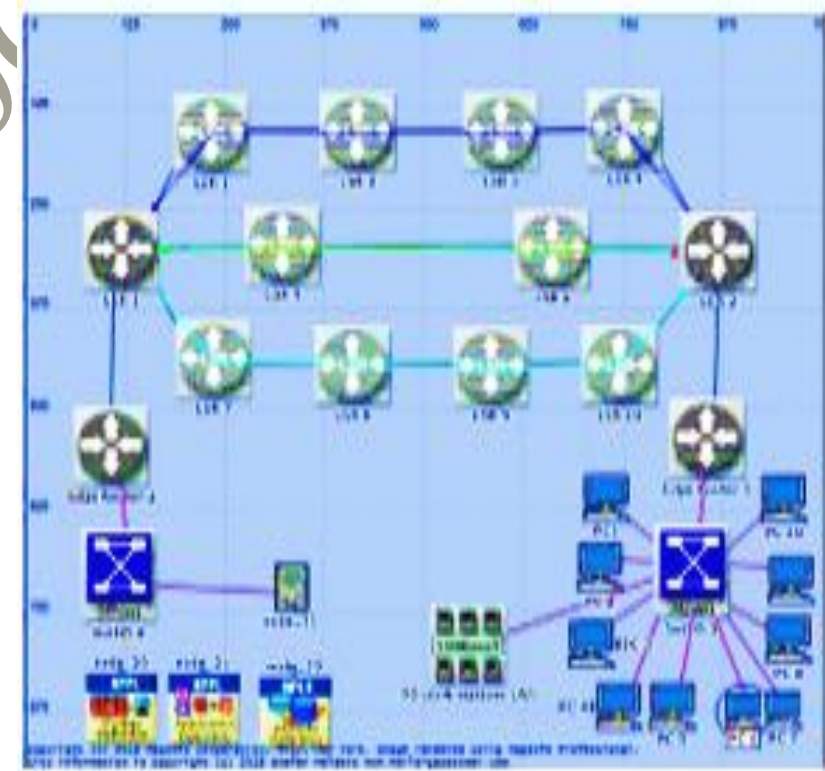

Fig (8) Layout of MPLS Simulation Model of Twelve Routers

In Figure 8, MPLS network is configured with twelve(12) core routers, LSP is configured in two ways, one from LER 2 to LER 1 through LSR 4,3,2,1 while the other through LSR 6, and 5. On the other hand, for the response of the request from switch 2 LSP are configured from LER 1 to LER 2 again from two ways one through LSR 5 and 6 while the second is through LSR 7,8,9,10. 


\section{SIMULATION RESULTS AND DISCUSSIONS}

All the simulation work is done by using the OPNET Modeler 14.5 network simulator. For each scenario in this research, there are totally three performance metrics are calculated to find the comparison of IP and MPLS networks in node scalable environments. These performance metrics are given below as:

\section{Average Packet drop / Traffic drop \\ Average End-to-End delay \\ Average Throughput}

Every scenario in this research is carefully run and has been investigated for three to four-time in a 1000x $1000 \mathrm{~m}$ Area and then the results are taken by using OPNET simulator graph. All results are shown an average value. On the behalf of these result then I have given my opinion about the performance of IP and MPLS networks in a node scalable environment.

Table 2 represents the overall outcome analysis of MPLS and IP on various scanarios. In table first column represents the number of routers utilized in this research, while other columns shows various ratio of Throughput, delay and packet loss for both MPLS and IP Network.

Table (2) MPLS and IP Network Performance Analysis on Various Scenarios

\begin{tabular}{|l|r|r|r|r|r|r|}
\hline \multirow{2}{*}{ No. R } & \multicolumn{3}{|c|}{ MPLS Network } & \multicolumn{3}{c|}{$\begin{array}{c}\text { Traditional IP } \\
\text { Network }\end{array}$} \\
\cline { 2 - 7 } & Th (\%) & $\begin{array}{c}\text { De } \\
\text { (sec) }\end{array}$ & PL & $\begin{array}{c}\text { Th } \\
\text { (b/s) }\end{array}$ & $\begin{array}{c}\text { De } \\
(\mathbf{s e c})\end{array}$ & PL \\
\hline 4 & $91 \%$ & 2 & $35 \%$ & $\begin{array}{r}81.20 \\
\%\end{array}$ & $\begin{array}{r}2.6 \\
\text { ) }\end{array}$ & $\begin{array}{r}48.33 \\
\%\end{array}$ \\
\hline 8 & $62 \%$ & 2.2 & $50 \%$ & $\begin{array}{r}37.50 \\
\%\end{array}$ & 3.8 & $55 \%$ \\
\hline 12 & $25 \%$ & 2.2 & $71 \%$ & 22.20 & 4 & $82 \%$ \\
\hline
\end{tabular}

here, NO. $\mathrm{R}=$ No of Routers, $\mathrm{Th}=$ Throughput, $\mathrm{De}=$ Delay, $\mathrm{PL}=$ Packet Loss

\section{A) Average Packet Drop / Traffic Drop}

Packet loss happens when one or more than one packet of data become fails to reach their destination. Packet loss mostly occurs when a network is congested.

In Figure 9 the bottom $x$-axis represents the number of routers for both MPLS and IP networks which is variable in each scenario. It's clearly noticed from the graph that when the number of routers is four (4), MPLS Packet drop ratio is $35 \%$ and this ratio is $48.33 \%$ in IP network, when these number of routers are increased, this packet loss rati also increased as 50\% in MPLS network and 55\% for IP network, when the network become more congested, as number of routers are increased from eight (8) to twelve (12) with end users increased for both technologies,packet loss ratio also start increasing to $71 \%$ for MPLS and $81 \%$ for IP networks. This is concluded that MPLS has a low packets loss ratio as compared to traditional IP networks even in scalable networks.

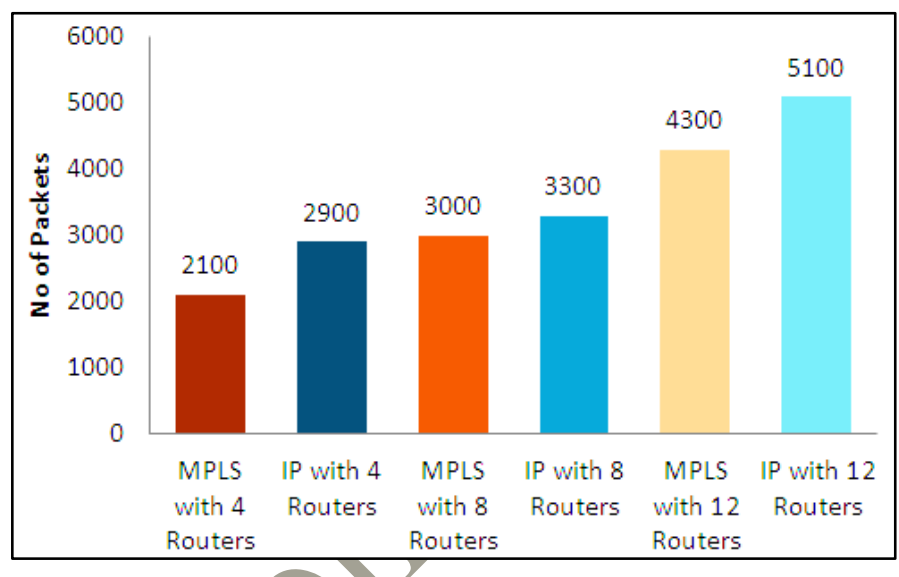

Fig (9) Comparison of Average Packet Loss of All Scenarios

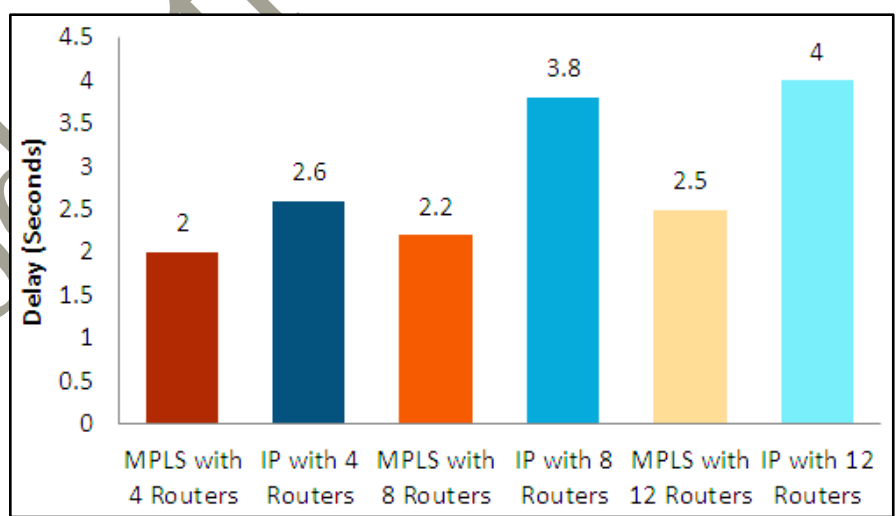

Fig (10) Comparison of Average Delay of All Scenarios

Figure 10 describes the comparison of delay for both MPLS and IP network for overall scenarios. It is noticed that when network is simple the network has minimum delay but as network become large, delay also gradually increased as we can see there is $2 \mathrm{sec}$ delay for MPLS and $2.6 \mathrm{sec}$ delay for IP network when number of routers is 4 , when number of routers is increased from four (4) to eight (8) the delay ratio also increased i.e 2.2 for MPLS network and 3.8 for IP network, when the routers are increased to twelve (12). its ratio gradually increased up to 4 for IP network and 2.5 for MPLS network which clearly shows that MPLS has low network delay in node scalable environment.

\section{B) Average Throughput}

Throughput is the number of various packets magnificently reached at destination per unit time. Throughput is represented in bits per seconds (bps) or packets per second. 


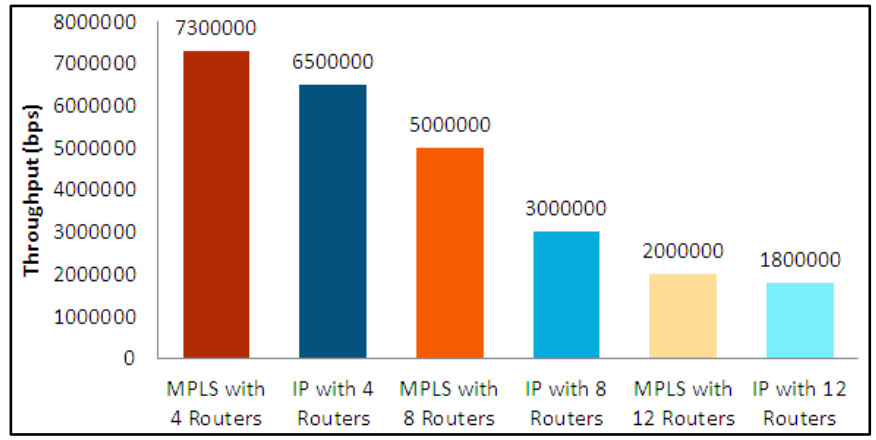

Fig (11) Comparison of Average Network Throughput of All Scenarios

In Figure 11 the vertical axis represents throughput in form of bits/sec. In the network of four (4) routers MPLS gives $91.25 \%$ throughput while IP network gives $81.25 \%$ throughput, as numbers of routers are increased from four to eight, network throughput starts decreasing which is $62.2 \%$ for MPLS network and $37.5 \%$ for IP network, also when number of routers increased from eight to twelve,throughput ratio also gets decrease as $25 \%$ while $22.2 \%$ for IP network. On the bases of this throughput ratio it can be clearly examined that MPLS has a good throughput than traditional IP network for multimedia traffic.

\section{CONCLUSION}

From the simulations carried out above and various experiments performed, this has been concluded that Internet Protocol network has lower performance as compared to that of MPLS technology.Multimedia applicatioions have much to concern about these two.The performance matrics concists of parameters like Packet Loss,End-to- End delay and Throughput while OPNET 14.5 modler was used for analyzing various network topologies. Simulations on modler were performed more then ten times and the conclusion, that was reached on, showed the fillowing facts and figures.

1. IP has more Packet Loss as compared to MPLS

2. IP has more Delay Time (end-to-end delay) as compared to MPLS

3. MPLS has more throuput then IP

The simulations also take us to the conclusion that ratio of packet loss and delay are slightly increased when the number of nodes increased in both technologies but this effect is lower in MPLS as compared to IP. Similarly, MPLS has much better throughput with increasing no of nodes as compared to IP for multimedia traffic. The values have been shown in table 1. This is concluded here, that,MPLS is much suited for heavy traffic multimedia data rather then IP technology.

\section{FUTURE WORK}

The future work of this research is to study and compare the IP and MPLS signaling protocol for implementing VoIP and multimedia application.

Improving of network throughput using MPLS traffic engineering is also future work of this research.

The comparison of IP and MPLS by changing the protocol and traffic type include in future work of this research.

The minimization of network delay and improving the network throughput is still a challenging task in future for researcher.

\section{REFERENCES}

[1] S. Veni, G. M. Kadhar Nawaz, and P. Praba, "Performance analysis of network traffic behavior in conventional network over MPLS," 2010 IEEE Int. Conf. Commun. Control Comput. Technol. ICCCCT 2010, pp. 222-226, 2010

[2] R. Shaker Naoum and M. Maswady, "Performance Evaluation for VOIP over IP and MPLS," World Comput. Sci, Inf. Technol. J., vol. 2, no. 3, pp. 110$114,2012$.

A3] P.,Ahlawat, "Comparison between Traditional IP Networks / Routing and MPLS," Int. J. Sci. Eng. Res., vol. 3, no. 3, 2015.

K. Jannu and R. Deekonda, "OPNET simulation of voice over MPLS,” no. June, 2010.

[5] M. Bhandure, "Comparative Analysis of Mpls and Non -Mpls Network," International Journal of Engineering Research and Applications, vol. 3, no. 4. pp. 71-76, 2013.

[6] L. He and P. Botham, "Pure MPLS technology," ARES 2008 - 3rd International Conference on Availability, Security, and Reliability, Proceedings. pp. 253-259, 2008.

[7] M. A. Rahman, A. H. Kabir, K. A. M. Lutfullah, M. Z. Hassan, and M. R. Amin, "Performance analysis and the study of the behavior of MPLS protocols," Proc. Int. Conf. Comput. Commun. Eng. 2008, ICCCE08 Glob. Links Hum. Dev., no. July, pp. 226229, 2008.

[8] R. Mishra and H. Ahmad, "Comparative Analysis of Conventional IP Network and MPLS Network over VoIP Application," vol. 5, no. 3, pp. 4496-4499, 2014.

[9] A. Sharma, R. Kumar, and V. Mansotra, "Proposed Stemming Algorithm for Hindi Information Retrieval," Int. J. Innov. Res. Comput. Commun. Eng. (An ISO Certif. Organ., vol. 3297, no. 6, pp. 11449-11455, 2016. 
[10] J. Tahir, M. Z. Siddiqi, and S. Arif, "Performance analysis of MPLS based networks with conventional networks," Proc. 2017 2nd Work. Recent Trends Telecommun. Res. RTTR 2017, pp. 17-20, 2017.

[11] A. Katiyar and P. K. Shrama, "OPTIMIZATION OF MPLS AND IP NETWORK IN VOICE,” no. 1, pp. 45-51, 2017.

[12] A.-B. R. Sulaiman and O. K. Salih Alhafidh, "Performance Analysis of Multimedia Traffic over MPLS Communication Networks with Traffic Engineering," Int. J. Comput. Networks Commun. Secur. Multiprotocol Label Switch. (MPLS), Traffic Eng., vol. 2, no. 3, pp. 93-101, 2014.

[13] J. F. Banu, K. G. Shanthi, P. L. Priya, and M. F. Begum, "Performance Enhancement Architecture of VoIP Applications in Multiprotocol Label Switching Networks," Circuits Syst., vol. 07, no. 08, pp. 20472058, 2016. 